\title{
Stability of Ca-montmorillonite hydrates: A computer simulation study
}

\author{
G. Odriozold A and J. F. Aguilari \\ Programa de Ingeniería Molecular, Instituto Mexicano del Petróleo, \\ Lázaro Cárdenas 152, 07730 México, Distrito Federal, México
}

(Dated: August 27, 2018)

\begin{abstract}
Classic simulations are used to study interlayer structure, swelling curves, and stability of Camontmorillonite hydrates. For this purpose, $N P_{z z} T$ and $\mu P_{z z} T$ ensembles are sampled for ground level and given burial conditions. For ground level conditions, a double layer hydrate having $15.0 \AA$ of basal spacing is the predominant state for relative vapor pressures $\left(p / p_{0}\right)$ ranging in $0.6-1.0$. A triple hydrate counting on $17.9 \AA$ of interlaminar distance was also found stable for $p / p_{0}=1.0$. For low vapor pressures, the system may produce a less hydrated but still double layer state with $13.5 \AA$ or even a single layer hydrate with $12.2 \AA$ of interlaminar distance. This depends on the established initial conditions. On the other hand, the effect of burial conditions is two sided. It was found that it enhances dehydration for all vapor pressures except for saturation, where swelling is promoted.
\end{abstract}

PACS numbers:

\section{INTRODUCTION}

Clay minerals are negatively charged layer type aluminosilicates kept together by cations. Since they constitute a great portion of soils and sedimentary rocks, their study impacts on podology, geology, geochemistry and ecology. Clays such as smectite group ${ }^{1}$ have the ability of absorbing water among clay sheets (interlayer spaces), in some cases producing a remarkable expansion of the mineral. This expansion is firstly crystalline (few water layers), and an osmotic regime is archived for higher water interlaminar contents 2 . The study of ions interacting with hydrated clay minerals is of particular interest, as they rule the swelling capacity of a given clay.

In engineered settings, clay mineral swelling is a critical factor in problems such as borehole stability in petroleum extraction, and in the liner and buffer stability in the containment of hazardous waste in geoenvironmental technology. One way to stabilize the shale is by reducing the swelling capacity of the clay by means of replacing sodium ions with divalent calcium ones through cation exchange methods $1.3,4.5$. Literature reports indicate that clays containing exchangeable calcium ions swell not as much as sodium clays, which are known to swell up to form water clay dispersions 1.5.6.7. This makes calcium chloride solutions the preferred internal phase of most oilbased drilling fluids. Additionally, calcium ions control water activity. This determines the osmotic movement of water between the drilling fluid and the formation, which may reduce the clay swelling when it is correctly handled. Thus, a detailed knowledge of the stability of the Ca-clay hydrates under different water activities is a key for an appropriate mud design.

As well as other Ca-montmorillonite simulation studies ${ }^{8.9}$, this work deals with the microscopic mechanisms underlaying Ca-montmorillonite swelling, but focussing on the stability of the different hydrates in contact with several reservoirs. These reservoirs differ in temperature, pressure, and composition (water activity). In order to fix these variables, the $\mu P_{z z} T$ ensemble is sampled, where $\mu$ refers to the water chemical potential of the reservoir. Since it is related to the relative partial water vapor pressure, plots of interlaminar distances and number of water molecules against it are constructed. For ground level conditions $(\mathrm{P}=1 \mathrm{~atm}$ and $\mathrm{T}=298 \mathrm{~K})$, these plots are directly accessed by experiments, making the comparison easy. Since good agreement was found, we expect the model to predict the behavior of the system for other non easily implementable experimental conditions. That is, for a given average burial depth. Hence, we studied the behavior of the model under $\mathrm{P}=600 \mathrm{~atm}$ and $\mathrm{T}=394 \mathrm{~K}, i$. e., for $4 \mathrm{~km}$ of depth assuming average gradients of $30 \mathrm{~K} / \mathrm{km}$ and $150 \mathrm{~atm} / \mathrm{km}$. We expect these data to be useful for developing drilling strategies.

This article is organized as follows. In Sec. III we briefly describe the models and the methodology employed for performing the simulations. The results are shown in Sec. III Finally, Sec. IV summarizes the main results and extracts some conclusions.

\section{METHODOLOGY}

\section{A. The model}

A montmorillonite clay simulation supercell was constructed by a $4 \times 2$ replication of the cell given by Skipper et al. $\stackrel{10}{.}$. The only difference is that in our model the octahedral oxygen sites have a charge of $-1.52 e$, and their corresponding hydrogen sites have a charge of $0.52 e$. This is for a better accordance with the TIP4P model ${ }^{11}$, which was employed for modelling water ${ }^{12}$. A Wyoming type montmorillonite was obtained by isomorphous substitutions of trivalent $\mathrm{Al}$ atoms of the octahedral sites by divalent $\mathrm{Mg}$ atoms, and tetravalent $\mathrm{Si}$ of the tetrahedral sites by trivalent $\mathrm{Al}$ atoms. The resulting layer counts on dimensions of $21.12 \times 18.28 \AA$ in the $(x, y)$ plane and a thickness of $6.56 \AA$. Water molecules were randomly distributed in the interlaminar spaces. The negative charge of the clay framework was 
TABLE I: Lennard-Jones parameters for water-clay interactions.

\begin{tabular}{ccc}
\hline \hline Sites & $\epsilon(\mathrm{kcal} / \mathrm{mol})$ & $\sigma(\AA)$ \\
\hline $\mathrm{O}$ & 0.1550 & 3.1536 \\
$\mathrm{H}$ & 0.0000 & 0.0000 \\
$\mathrm{Si}$ & 3.150 & 1.840 \\
$\mathrm{Al}$ & 3.150 & 1.840 \\
$\mathrm{Mg}$ & 3.150 & 1.840 \\
\hline \hline
\end{tabular}

balanced by three calcium ions placed in the interlayer midplanes. The resulting unit cell formula is given by $\mathrm{Ca}_{0.375} n \mathrm{H}_{2} \mathrm{O}\left(\mathrm{Si}_{7.75} \mathrm{Al}_{0.25}\right)\left(\mathrm{Al}_{3.5} \mathrm{Mg}_{0.5}\right) \mathrm{O}_{20}(\mathrm{OH})_{4}{ }^{\frac{8}{}}$.

The water-clay interactions are taken from Boek ${ }^{13}$ et al. Here, the total interaction potential is given by a Coulombian plus a Lennard-Jones contribution,

$$
U_{i j}=\sum_{a, b}\left[\frac{q_{a} q_{b}}{r_{a b}}+4 \epsilon_{a b}\left[\left(\frac{\sigma_{a b}}{r_{a b}}\right)^{12}-\left(\frac{\sigma_{a b}}{r_{a b}}\right)^{6}\right]\right]
$$

where subindexes $i$ and $j$ are for molecules, and $a$ and $b$ run over all sites of each molecule. $q_{a}$ and $q_{b}$ are the corresponding site charges, $\epsilon_{a b}$ and $\sigma_{a b}$ are site-to-site specific Lennard-Jones parameters, and $r_{a b}$ is the intersite distance. The site-to-site Lennard-Jones parameters are given by the Lorentz-Berthelot rules

$$
\begin{gathered}
\sigma_{a b}=\frac{\sigma_{a}+\sigma_{b}}{2}, \\
\epsilon_{a b}=\sqrt{\epsilon_{a} \epsilon_{b}}
\end{gathered}
$$

The corresponding Lennard-Jones parameters for different species are given in Table【 Parameters for Si were taken from Marry et al $\stackrel{14}{\underline{4}}$, and parameters for $\mathrm{Al}$ and $\mathrm{Mg}$ were assumed to be equal to those of $\mathrm{Si}$.

The Ca-O and $\mathrm{Ca}-\mathrm{H}$ interactions are based on the ones proposed by Bounds $\frac{15}{}$, since they produce Ca-TIP4P radial distribution functions in agreement with available experimental data and close to hybrid quantum mechanics/ molecular mechanics (QM/MM) results. That is, the $\mathrm{Ca}-\mathrm{O}$ radial distribution function peaks at $2.54 \AA$ leading to a first shell oxygen coordination number of 9.3 , while the experimental results are close to $2,46 \AA^{16}$ and a wide range of coordination numbers turn into 6.010.016.17.18.19. On the other hand, hybrid QM/MM simulations performed at DFT level (LANL2DZ basis sets) lead to $2.51 \AA$ of $\mathrm{Ca}-\mathrm{O}$ distance and a coordination number of $8.1^{20}$. The pair potential reads

$$
\begin{aligned}
U_{\mathrm{Ca}-\mathrm{H}_{2} \mathrm{O}}= & A_{\mathrm{CaO}} \exp \left(-b_{\mathrm{CaO}} r_{\mathrm{CaO}}\right)-C_{\mathrm{CaO}} / r_{\mathrm{CaO}}^{4} \\
& -D_{\mathrm{CaO}} / r_{\mathrm{CaO}}^{6}+A_{\mathrm{CaH}} \exp \left(-b_{\mathrm{CaH}} r_{\mathrm{CaH}}\right) \\
& +A_{\mathrm{CaH}} \exp \left(-b_{\mathrm{CaH}} r_{\mathrm{CaH}}\right),
\end{aligned}
$$

with $A_{C a O}=37146.0 \mathrm{kcal} \mathrm{mol}^{-1}, b_{C a O}=2.9902 \AA^{-1}$, $C_{C a O}=1578.6 \mathrm{kcal} \AA^{4} \mathrm{~mol}^{-1}, D_{C a O}=-2185.3 \mathrm{kcal} \AA^{6}$ $\mathrm{mol}^{-1}, A_{C a H}=8212.0 \mathrm{kcal} \mathrm{mol}^{-1}$ and $b_{C a H}=3.7234$ $\AA^{-1}$.

Since it is crucial for the hybrid Monte Carlo (HMC) simulations to keep the energy fluctuations as low as possible in order to enlarge the acceptation rate ${ }^{21}$, it is convenient to avoid employing relatively long range pair potential contributions, such as $\sim r^{-4}$, if no Ewald treatment is applied on them. Thus, we refitted to equation 4 the following expression

$$
\begin{aligned}
U_{\mathrm{Ca}-\mathrm{H}_{2} \mathrm{O}}= & A_{\mathrm{CaO}} \exp \left(-b_{\mathrm{CaO}} r_{\mathrm{CaO}}\right)-C_{\mathrm{CaO}} / r_{\mathrm{CaO}}^{6} \\
& +A_{\mathrm{CaH}} \exp \left(-b_{\mathrm{CaH}} r_{\mathrm{CaH}}\right) \\
& +A_{\mathrm{CaH}} \exp \left(-b_{\mathrm{CaH}} r_{\mathrm{CaH}_{2}}\right),
\end{aligned}
$$

by employing a Levenberg-Marquardt algorithm, considering several $\mathrm{Ca}-\mathrm{H}_{2} \mathrm{O}$ configurations. The procedure yields $A_{C a O}=229184.5 \mathrm{kcal} \mathrm{mol}^{-1}, b_{C a O}=3.3626 \AA^{-1}$, $C_{C a O}=15616.5 \mathrm{kcal} \AA^{6} \mathrm{~mol}^{-1}, A_{C a H}=2417.0 \mathrm{kcal}$ $\mathrm{mol}^{-1}$ and $b_{C a H}=3.0382 \AA^{-1}$. We observed that by avoiding the $\sim r^{-4}$ term, the acceptance rate enlarges more than three times for a small (inner) time step of $8 \times 10^{-4} \mathrm{ps}$, this being the typical value. To check the reliability of this Bound based Ca-water potential, NPT simulations with 216 water molecules, a calcium cation, and two chloride anions were performed. From the radial distribution functions we observed that the first and second oxygen shells are situated at 2.53 and $4.43 \AA$ respectively, while for $\mathrm{Ca}-\mathrm{H}$ they are at 3.05 and $5.05 \AA$. The coordination number for the first oxygen coordination shell is 8.75. These values are in agreement with experimental data and are casually closer than Bound's potential results to those obtained by Schwenk et al ${ }^{20}$ Additionally, results also compare well with those reported by D. G. Bounds $\frac{15}{}$. Therefore, expression 5 seems suitable for describing the Ca-TIP4P interactions.

Periodic boundary conditions were imposed on the three space directions. The electrostatic interactions $\sim r^{-1}$ are computed using the Ewald summation method, and a spherical cutoff equal to half the smallest box side is set for the short range interactions. Standard corrections for the short range interactions were considered ${ }^{22}$.

\section{B. Simulations}

Our simulation methodology is based on previous work $^{23}$ thus it is not discussed in detail here. The simulations were performed employing a HMC method 21.23 . A reversible multiple time scale algorithm ${ }^{24}$ is employed as discretization scheme. The long time step is set up to 8 times the short time step, and the short time step is chosen to obtain an average acceptance probability of $0.7^{21}$. To keep time correlations as low as possible, a new configuration is generated each 10 integration steps. The probability to accept this new configuration is

$$
P=\min \{1, \exp (-\beta \Delta \mathcal{H})\}
$$




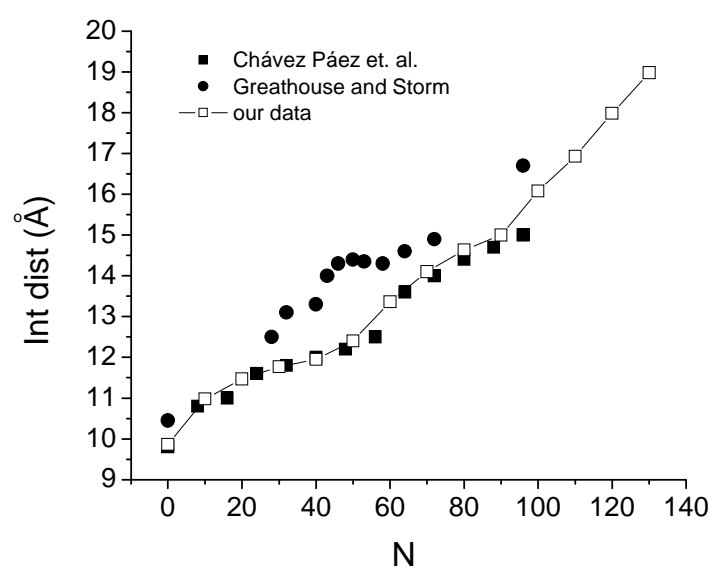

FIG. 1: Interlaminar distance as a function of the number of water molecules per interlaminar space for ground level conditions.

where $\Delta \mathcal{H}$ is the difference between the new and previous configuration Hamiltonians, and $\beta$ is the inverse of the thermal energy.

For sampling in the $N P_{z z} T$ ensemble the stress normal to the surface of the clays, $P_{z z}$, is kept constant. Thus, only volume fluctuations in the $z$-direction are allowed, and the probability for accepting a new box configuration is given by

$$
P=\min \left\{1, \exp \left[-\beta\left(\Delta \mathcal{U}+P_{z z} \Delta V-N \beta^{-1} \ln \left(V_{n} / V_{o}\right)\right)\right]\right\}
$$

Here, $\Delta \mathcal{U}$ is the change in the potential energy, $\Delta V$ is the volume change, $N$ is the total number of molecules, and $V_{n}$ and $V_{o}$ are the new and old box volumes, respectively 25 .

The $\mu P_{z z} T$ ensemble is sampled by simply performing particle movements, insertions and deletions, and box changes as in typical $N V T, \mu V T$, and $N P_{z z} T$ samplings ${ }^{23}$. Water insertions and deletions were performed by Rosenbluth sampling 23.26. Different types of movements are called just like explained elsewhere ${ }^{23}$. This algorithm provides a good way of sampling this kind of systems, since only one run is necessary to obtain the equilibrium state at reservoir conditions. This contrasts with the more frequently used $\mu V T$ sampling, that leads to similar information after a large number of simulation runs 8.25 .26 .27 .28 . It should be also mentioned that in typical x-ray experiments the ambient vapor pressure, the total pressure, and the temperature are controlled, but not the water content of the interlaminar space or the interlaminar distance. These are exactly the same variables that are setup in a $\mu P_{z z} T$ simulation, which consequently allows for a fair comparison. On the other hand, $N P_{z z} T$ and $\mu V T$ ensembles may force the system to pass trough equilibrium states that are not produced in real experiments. This is the case of water contents equivalent to 55 water molecules per interlaminar space or interlaminar distances of $13 \AA$ for sodium montmorillonite, since they do not correspond to a single or to a

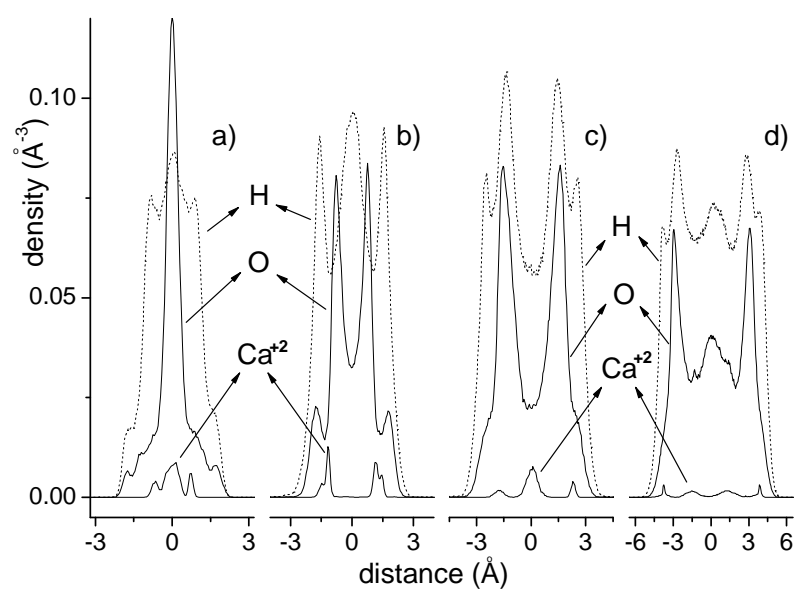

FIG. 2: Oxygen, hydrogen and calcium density profiles of the interlaminar space for ground conditions. The water amount was fixed to 40, 60, 90 and 120 molecules per interlaminar space, from left to right, respectively.

double water hydrate. Nevertheless, one may artificially produce these states by simply fixing $N$ or $V$ in $N P_{z z} T$ or $\mu V T$ sampling. Measuring the water chemical potential for the first case ${ }^{29}$, or the pressure for the second ${ }^{25}$, large values of chemical potential and pressure are obtained. In fact, these variables show oscillations when obtained as a function of $N$ or $V$, indicating that several equilibrium states are possible $e^{25.29}$. These measurements also suggest the existence of energetic barriers that separates the single, double, and triple layer hydrates. Since in $\mu P_{z z} T$ sampling the interlaminar distance and the number of water molecules are free variables, these states are simply avoided, splitting the phase space in two (or more) regions. For these cases, two (or more) different equilibrium states appear, which are accessed by handling initial conditions. This way, hysteresis cycles are naturally obtained.

\section{RESULTS}

The swelling curve obtained for ground level conditions, i. e. $\mathrm{P}=1 \mathrm{~atm}$ and $\mathrm{T}=298 \mathrm{~K}$, is shown in figure [1 Here, each data point is obtained by performing a $N P_{z z} T$ simulation and hence, by fixing the number of water molecules. This figure also includes the data reported by Chávez-Páez et al $\stackrel{25}{n}^{25}$ and by Greathouse and Storm ${ }^{9}$. As can be seen, our data compare very well with those of Chávez-Páez et al. Since methodologies and models are similar this is something expected. Larger differences are seen between our data and those reported by Greathouse and Storm, who employed the Lennard-Jones type Ca-O potential given by Aquist ${ }^{30}$. These differences point out to a general weakness associated with the use of classical force fields, which may be overcome by employing ab initio molecular dynamics simulations. This was successfully done by Boek et al $\underline{31}^{31}$ 
Systems counting on 40, 60, 90 and 120 water molecules (wm) per interlaminar space produce 12.0, 13.4, 15.0, and $17.9 \AA$ of interlaminar distance. The first one corresponds to a single water layer formation. The last two are frequently observed in experiments and should correspond to double and triple water layer hydrates, while the interlaminar distance of 13.4 $\AA$ is sometimes obtained experimentally for small vapor pressures 32.33 .34 . The corresponding oxygen, hydrogen, and calcium profiles are shown in figure 2 Oxygen peaks of this figure make clear that the structures correspond to a single water layer hydrate, to two double layer hydrates, and to a three layer hydrate, from left to right. It also shows that the double layers structures differ on their hydrogen and calcium profiles. These calcium profiles pass from two double peaks located close to the clay layers, suggesting the formation of different types of inner-sphere complexes, to a structure having a very important middle peak, indicating the formation of outer-sphere complexes. This tendency for calcium ions to detach from the surface with increasing water content agree with the predictions of Greathouse and Storm ${ }^{9}$.

To confirm the presence of inner and outer-sphere complexes some snapshots were analyzed. From them figure 3] was built, where only those water molecules having Ca$\mathrm{O}$ distances smaller than $3.0 \AA$ are shown. Two innersphere complexes are seen, [3 a) and b), and an outersphere complex, 3 c). The complex shown in figure 3 a) has no tetrahedral substitution involved, whereas in figure 3 b) the calcium ion is attached to a tetrahedral substitution. As can be seen, two oxygens coordinate with the ion for the first case, and the three surrounding the aluminium atom in the second. In both cases, $6 \mathrm{wm}$ complete the inner sphere shell. In addition, distances from clay oxygens are much smaller when the tetrahedral substitution is involved. These different coordination distances explain the calcium double peaks seen in figure 2 b). On the other hand, $8 \mathrm{wm}$ form the inner sphere shell in case of outer-sphere complexes, in agreement with simulations ${ }^{9}$ and experimental evidence ${ }^{35}$. For double layer hydrates, these complexes are situated close to the interlayer midplane, leading to $\mathrm{Ca}-\mathrm{O}$ distances for clay oxygens in the range of 4.3-5.0 $\AA$ (these distances are not highlighted in the figure). It should be noted that they are similar to the $\mathrm{Ca}-\mathrm{O}$ distances found for the water second shell that surrounds calcium in bulk water. Hence, this may enhance the double layer stability.

In order to build swelling curves as a function of the water vapor pressure of a reservoir in contact with the system, the $\mu P_{z z} T$ ensemble was sampled. The relationship between the chemical potential and the vapor pressure is $\beta \mu=\beta \mu_{0}+\ln \left(p / p_{0}\right)$, where $p_{0}$ is the vapor pressure at equilibrium with liquid water whose chemical potential is $\mu_{0}$, and $p$ is the vapor pressure. For the TIP4P model, $1 \mathrm{~atm}$, and $298 \mathrm{~K}$, we employed $\beta \mu_{0}=-$ $17.4^{23,25}$. In case of $600 \mathrm{~atm}$ and $394 \mathrm{~K}$ the employed value is $-13.4^{23}$.

A sampling example from the $\mu P_{z z} T$ ensemble is shown
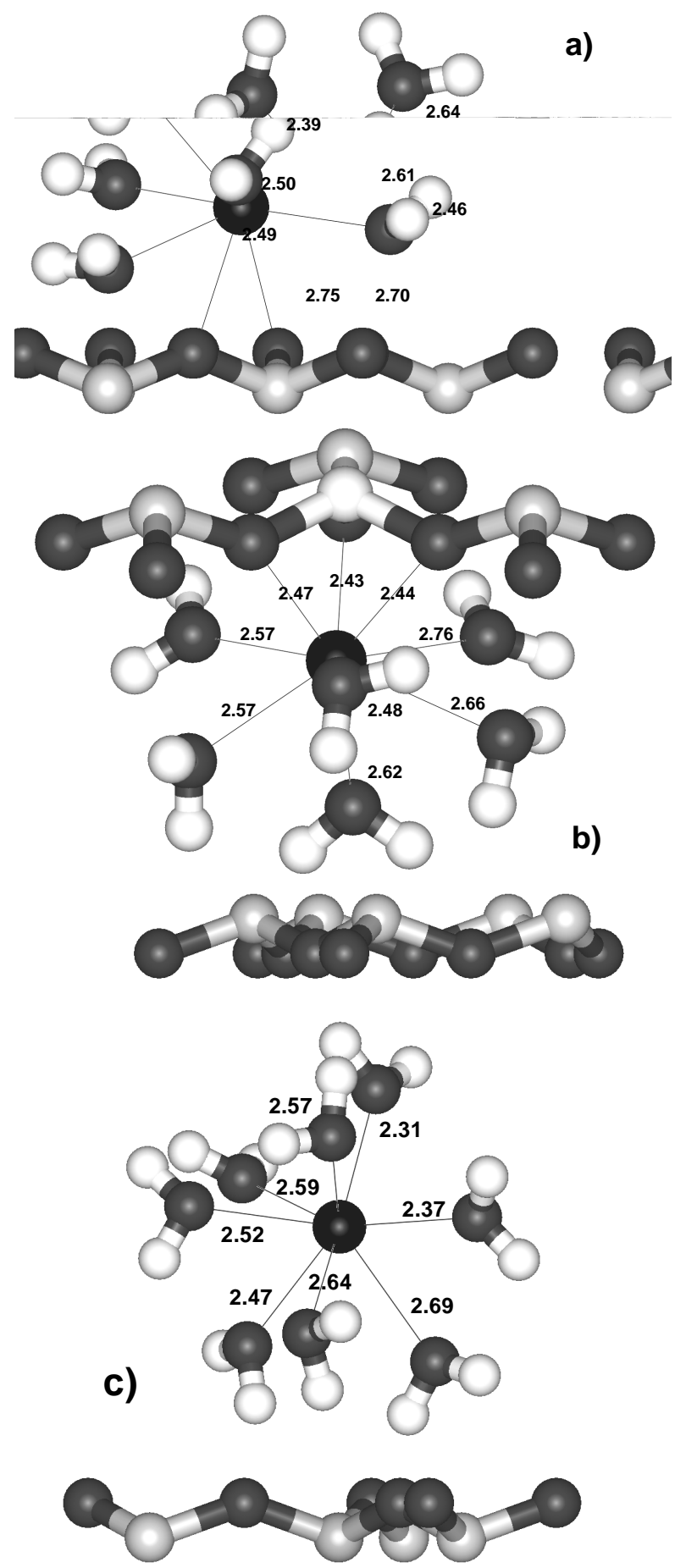

FIG. 3: Zoom in of calcium ions (black) obtained from equilibrium configurations. Only water molecules counting on $\mathrm{Ca}-\mathrm{O}$ distances smaller than $3.0 \AA$ are shown. $\mathrm{H}$ are represented by white, $\mathrm{O}$ by dark-gray, and $\mathrm{Si}$ atoms by lightgray spheres. Distances in the figure are given in $\AA$. a) $\mathrm{Ca}^{++}$ forming an inner-sphere surface complex. b) $\mathrm{Ca}^{++}$forming an inner-sphere surface complex that involves a tetrahedral substitution of a silicon by an aluminium (white), and c) $\mathrm{Ca}^{++}$ forming an outer-sphere complex. 


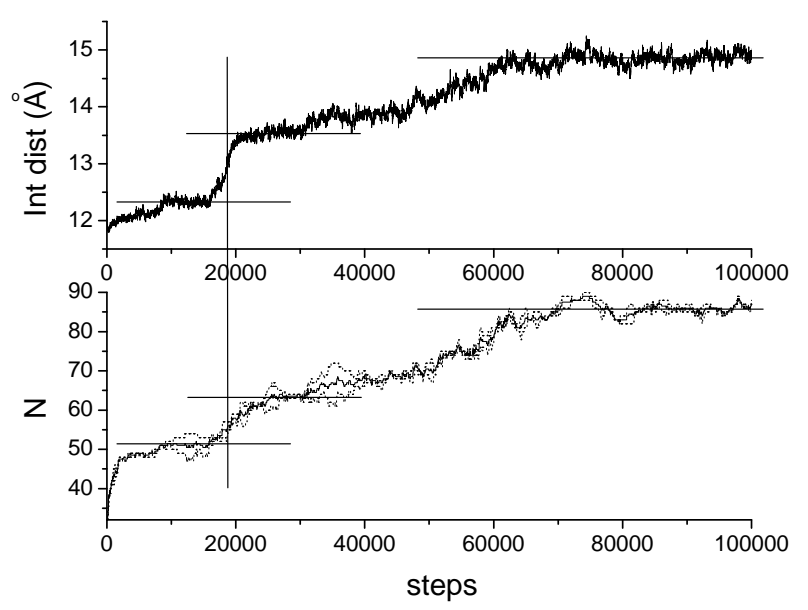

FIG. 4: Evolution of the interlaminar distance and water content with the number of simulation steps. For the lower plot, dotted lines are the water content for each interlaminar space and the solid line is the average. Initial conditions are close to the dehydrated state, $\mathrm{p}=0.8 p_{0}$, and ground level conditions were imposed. Horizontal and vertical lines are just a guide to the eye.

in figure 4 Here, the interlaminar distance, the water amount of each interlaminar space, and the average amount of water are plotted against the simulation step. For this particular case the simulation was started from an almost dehydrated state in contact with a reservoir having $p=0.8 p_{0}$, and for ground level conditions. It is seen how water molecules enter the system thickening the interlaminar distance. It is also noticed that once the system yields some of the interlaminar structures shown in figure 2 it shows certain resistance to swell to another state. For example, the resistance at $12.4 \AA$, corresponding to a single water layer hydrate, is overcome once the simulation has spent close to 8000 steps. Immediately after overcoming this resistance, the interlaminar distance jumps from 12.4 to $13.5 \AA$. This means that the interlaminar space rearranges from the single water layer shown in figure 2 a) to the double water layer corresponding to figure 2 b). Clearly, this rearrangement implies overcoming a sort of collective potential barrier. Something similar happens with the transition from the double layer hydrate at $13.5 \AA$ towards the structure shown in figure 2 c), although the rearrangement seems to be gradual. Here, most ions leave the surface to locate close to the interlaminar midplane. Finally, the system yields a stable state close to $15.0 \AA$ (stable at least for the finite number of considered steps, but confirmed by other runs having starting configurations closer to this final state).

We consider that the resistances found for rearranging the interlaminar structures point to local free energy minima, and their relative depths under the given conditions should be directly related to the number of steps the simulation spends on them. However, due to the stochastic character of this number, several runs should

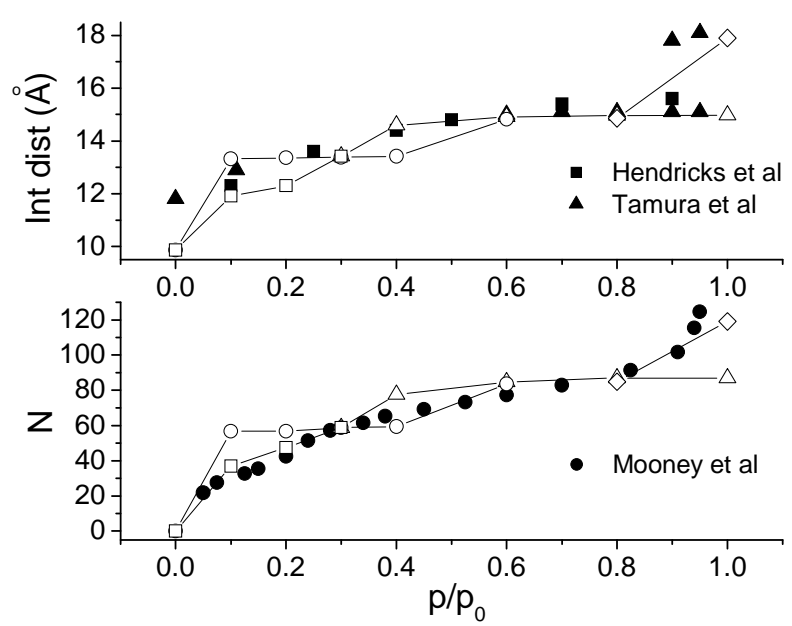

FIG. 5: Interlaminar distance and number of water molecules per interlaminar space as a function of the vapor pressure for ground level conditions. Symbols $\square, \circ, \triangle$, and $\diamond$ correspond to initial conditions of $10 \mathrm{wm}-12.0 \AA$, $60 \mathrm{wm}-15.0 \AA$, $90 \mathrm{wm}$ - $16.0 \AA$, and $120 \mathrm{wm}$ and $18.5 \AA$ of interlaminar distance, respectively.

be performed to obtain some reliable averages.

The swelling curves for ground level conditions are shown in figure 5 To generate them, four starting configurations were considered. These are: an almost dehydrated state having $10 \mathrm{wm}$ and $12.0 \AA$ of interlaminar space, $60 \mathrm{wm}-15.0 \AA$, $90 \mathrm{wm}-16.0 \AA$, and $120 \mathrm{wm}$ $18.5 \AA$ of interlaminar space. These configurations produce single, double, and triple layer hydrates in a few simulation steps. If the fixed conditions are consistent with these states, both, water content and interlaminar distance just fluctuate around certain mean values. On the contrary, if vapor pressure is not consistent with the hydrate, this is destabilized, producing another number of water layers. This is the case shown in figure 4 Limiting cases are the dehydrated state (no water molecules remain in the system) and the fully hydrated state (where the simulation leads to an ever increasing number of water molecules).

As expected, independently of the initial conditions, the simulations for $p=0$ yield the dehydrated state, counting on an interlaminar distance of $9.9 \AA$, in agreement with the $N P_{z z} T$ results. On the other hand, and also no mattering what the established initial conditions are, for $p \geq 0.6 p_{0}$ a double layer hydrate is always obtained, except for a saturated vapor pressure where a triple layer hydrate is also possible. The structure of this double layer hydrate is consistent with that shown in figure 2 c). It has a water content close to $87 \mathrm{wm}$ and an interlaminar space about $15.0 \AA$. On the other hand, the triple layer hydrate structure looks like that shown in figure 2 d) and has $17.9 \AA$ of interlaminar space and close to 119 wm.

For vapor pressures ranging in $0.1-0.4 p_{0}$, things are more complicated. Here, two equilibrium states were ob- 
served for $p=0.4,0.2$, and $0.1 p_{0}$. These are: two different double layer hydrates for $p=0.4 p_{0}$; and a single and a double layer hydrates for $p=0.2$ and $0.1 p_{0}$. These two double layer hydrates are those shown in figure 2 b) and c). As already mentioned, they differ on their water content and on their water-ion structure. The single hydrate shows interlaminar distances close to $12.2 \AA$. This hydrate was found stable just in the range of $0.1-0.2 p_{0}$, and the double layer hydrate with the smallest interlaminar distance is stable for $p=0.1-0.4 p_{0}$. This completes the description for the swelling of Ca-montmorillonite hydrates, showing two closed hysteresis loops for small water vapor pressures.

The obtained data are in good agreement with experimental results. In order to clearly see it, figure 5 includes the experimental data obtained by Hendricks et al . $^{33}$, Tamura et al $\underline{36}^{36}$ and Mooney et al $\underline{32}^{32}$. Here, not only the interlaminar distances for the single, double, and triple hydrates are reasonably matched but also their relative vapor pressure range. For example, the experimental basal spacing distances for the single layer hydrate range in $11.9-12.5 \AA ; 15.0-15.2 \AA$ are the values reported for the double layer hydrate; and 17.7-18.1 $\AA$ are those reported for the triple hydrate ${ }^{32.36 .37}$. Moreover, they even seem to support the interlaminar distances obtained for the double layer hydrate shown in figure 2 b). Therefore, values that range in 12.8-13.8 $\AA$ for small water vapor pressures 32.33 .34 may correspond to our double layer of $60 \mathrm{wm}$ per interlaminar space.

The number of water molecules contained in the system also match the data given by Mooney et al. . $^{32}$. We should mention here that for converting their data from water content to number of water molecules, it was assumed that $96 \mathrm{wm}$ correspond to $300 \mathrm{mg} \mathrm{H}_{2} \mathrm{O} / \mathrm{g}$ clay 9.12 .38 . The data plotted in figure 5 under the label Mooney et al. were obtained this way. As can be seen, the agreement is remarkable.

The swelling curve obtained for burial conditions and by means of $N P_{z z} T$ sampling is shown in figure 6 We also include the one obtained for ground conditions, for comparison. It is seen that for small water contents, both curves almost coincide. As water content increases, the curve obtained for burial conditions produces larger interlaminar spaces. Another effect is that the first plateau shortens, and a double layer formation is observed for water contents as low as $50 \mathrm{wm}$. Here, the first double layer plateau ranges in 50-60 wm, and there seems to be a second in the range 70-80 wm. This plateau displacement is due to the large effective volume the water molecules occupy for burial depth, being just a consequence of the larger temperature 29 .

The structures of the systems that correspond to the different plateaus are similar to those already shown in figure 2 The main difference is that profile peaks widen and shorten due to higher thermal energy. This was also observed by means of experiments 39 . Something similar happens with the $\mathrm{Ca}-\mathrm{O}$ radial distribution functions, although the first shell coordination numbers remain quite

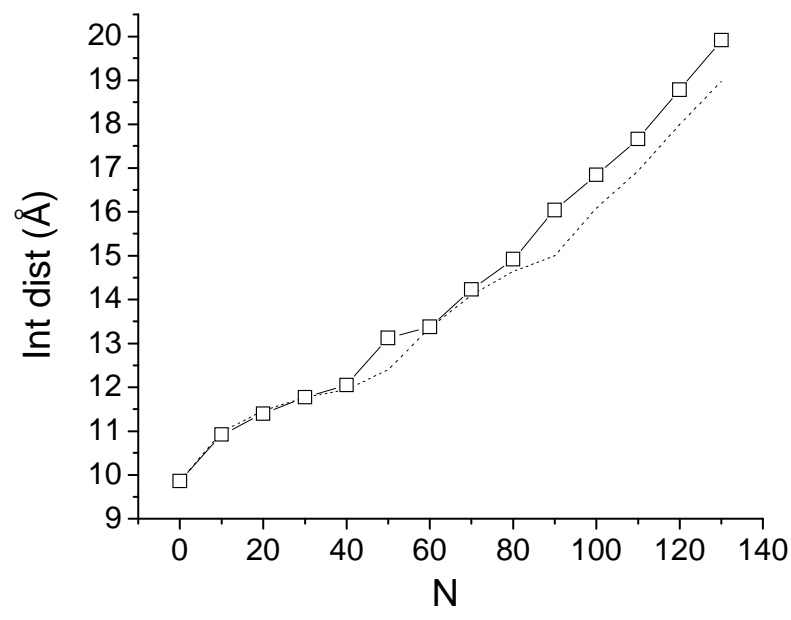

FIG. 6: Interlaminar distance as a function of the number of water molecules per interlaminar space for burial conditions. For comparison, the dotted line corresponds to ground conditions (from figure 1).

the same.

Figure 7 shows the swelling curves obtained for burial conditions. Just like figure 5 the water amount is not fixed since sampling was performed in the $\mu P_{z z} T$ ensemble. Therefore, in general, several different equilibrium states appear as a function of the imposed reservoir's vapor pressure, which are accessed by handling initial conditions. Figure 7 also includes the results for ground level conditions with dotted lines, for an easier comparison. To begin with, let us focus on the data obtained by starting from an almost dehydrated state. As expected, the full dehydration is only obtained for $p=0$. This has a very similar interlaminar distance than the one obtained for ground level conditions. For $0.1 p_{0} \leq p \leq 0.3 p_{0}$, the single water hydrate is yielded. This contrasts with the vapor pressure range obtained for the single hydrate at ground level conditions, which is shorter. This suggests that dehydration is favored for burial conditions. This hydrate counts on an interlaminar distance similar to that obtained for ground level conditions, although with a smaller water content. Again, this points towards the larger effective volume occupied by water molecules at higher temperatures. It is also seen that for a vapor pressure of $p=0.4 p_{0}$, the system produces a double layer hydrate having $13.3 \AA$ of interlaminar distance and 58 wm. This signatures the end of the single water layer domain.

The results for an initial condition of $60 \mathrm{wm}$ and $15.0 \AA$ of interlaminar distance are as follows. For $p=0.1-0.2 p_{0}$ the double layer hydrate is destabilized and a single layer hydrate is produced. In the range $0.3 p_{0} \leq p \leq 0.6 p_{0}$, this initial condition leads to a double layer hydrate similar to that shown in figure 2 b). This state has an interlaminar distance close to $13.3 \AA$ and a water content of $58 \mathrm{wm}$. Our data indicate that for $p=0.4$ and $0.6 p_{0}$ it turns into the only stable state. Hence, for $p=0.6 p_{0}$ the structure 


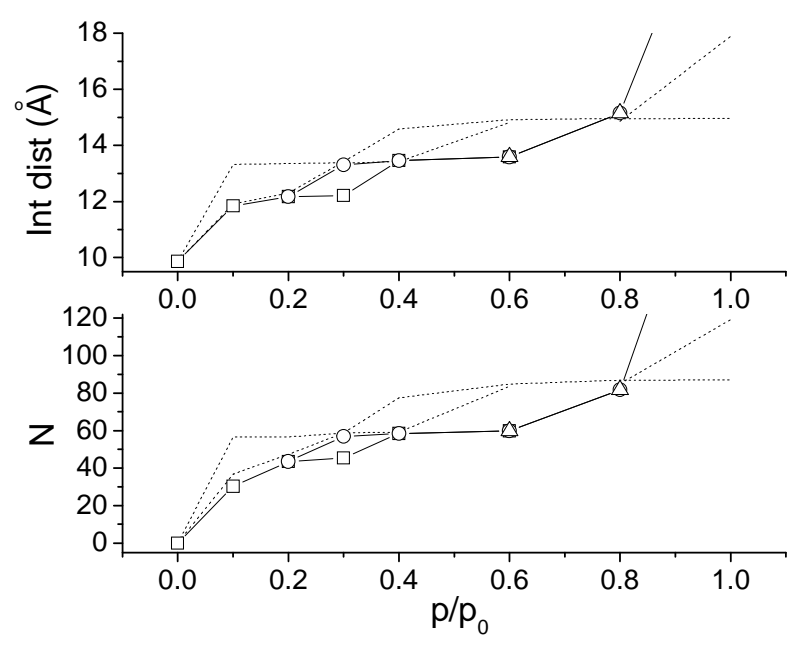

FIG. 7: Interlaminar distance and number of water molecules per interlaminar space as a function of the vapor pressure for burial conditions. Symbols $\square, \circ$ and $\Delta$ correspond to initial conditions of $10 \mathrm{wm}-12.0 \AA, 60 \mathrm{wm}-15.0 \AA$, and $90 \mathrm{wm}$ $16.0 \AA$ of interlaminar distance, respectively.

2] c) obtained under ground level conditions is also destabilized in favor of the structure $2 \mathrm{~b}$ ). All this points to a dehydration process occurring at burial depths as well.

The initial condition of $90 \mathrm{wm}$ and $16.0 \AA$ of interlaminar distance produces the double layer hydrate having $15.0 \AA$ of interlaminar distance only for $p=0.8 p_{0}$. For a saturated vapor pressure the hydrate becomes unstable and a full hydration is observed. On the other hand, it dehydrates for $p=0.6 p_{0}$. Therefore, it seems that burial conditions favor dehydration for all vapor pressures except for saturation, that, on the contrary, promotes swelling.

\section{CONCLUSIONS}

Ca-montmorillonite hydrates were studied by means of $N P_{z z} T$ and $\mu P_{z z} T$ simulations. Interlaminar structures and swelling curves for a fixed amount of water were analyzed by $N P_{z z} T$ sampling, whereas the $\mu P_{z z} T$ ensemble was used to build swelling curves as a function of the reservoir relative vapor pressure. Both ground level and burial conditions were considered.

Results indicate that under ground level conditions four interlayer structures are possible. For small relative vapor pressures, a single or a double layer hydrate with a low water content are obtained, counting on 12.2 and $13.4 \AA$ of interlaminar distance, respectively. For larger relative vapor pressures, a double hydrate with a higher water content and $15.0 \AA$ of interlaminar distance is obtained. Finally, a three layer hydrate with $17.9 \AA$ of interlaminar distance was detected for water vapor saturation. All these data well agree with experimental results.

It was observed that the more hydrated the system becomes, more ions fully hydrate to form outer-sphere complexes. In other words, inner-sphere complexes are mostly observed for low water contents. It should be mentioned that these outer-sphere complexes in double layer hydrates present part of their second water shell substituted by oxygen atoms of the two adjacent clay layers. This aids to counterbalance the expanding pressure the water molecules exert on the clay sheets. On the other hand, calcium ion lowers the interlaminar space water activity, favoring the entrance of water thus producing denser systems. This causes higher expanding pressures to deal with. Hence, it was not a priory clear if calcium ions were going to produce stable hydrates under all environments.

Burial conditions enhance dehydration for all vapor pressures except for saturation. This last case was found to provoke swelling. We should mentioned that a real reservoir with a saturated vapor pressure is not likely to occur, since there are always dissociated electrolytes that lower water activity. Thus, one can not expect this extreme case to happen in a real reservoir. For example, Wang et al $\stackrel{40}{n}$ were able to relate the dehydration temperature of montmorillonite in calcium solutions with its water activity, finding that relatively small quantities of calcium chloride produce a large enough drop of the vapor pressure to considerably lower dehydration temperature. In fact, this explains the extensive use of calcium chloride as the internal phase of oil-based drilling fluids. From figure 7 it is concluded that a drop of vapor pressure not only prevents swelling but also favors dehydration, indeed. Therefore, we consider that the most important role of calcium ion as swelling inhibitor is just to lower the water activity of the reservoir in contact with the montmorillonite hydrate system.

\section{ACKNOWLEDGMENTS}

This research was supported by Instituto Mexicano del Petróleo grant D.00072.
* godriozo@imp.mx

† aguilarf@imp.mx

1 K. Norrish, Discussions of the Faraday Society 18, 120 (1954).

2 H. van Olphen, An introduction to clay colloid chemistry
(Jhon Wiley and Sons, New York, 1977), 2nd ed.

3 C. E. Marshall, The colloid chemistry of the silicate minerals (Academic Press, New York, 1949).

${ }^{4}$ D. McEwan and J. J. Wilson, in Crystal structures of clay minerals and their $x$-ray identification, edited by 
G.W.Brindley and G.Brown (Mineralogical Society of London, London, 1980).

${ }^{5} \mathrm{H}$. van Olphen, in Chemistry of clays and clay minerals., edited by A.C.D.Newman (Mineralogical Society of London monograph 6, New York: John Wiley and Sons, 1987).

6 S. Chatterji, P. Christensen, and G. Overgaard, in Proceedings of the 3rd International Congress on the Deterioration and Preservation of Stone (Universita des li studi di Padoua, Venice, 1979).

7 A. Sridharan and P. V. Satyamurty, Clays and Clay Miner. 44, 479 (1996).

8 M. Chávez-Páez, L. de Pablo, and J. J. de Pablo, J. Chem. Phys. 114, 10948 (2001).

9 J. A. Greathouse and E. W. Storm, Mol. Sim. 28, 633 (2002).

10 N. T. Skipper, F. Chou Chang, and G. Sposito, Clays and Clay Miner. 43, 285 (1995).

11 W. L. Jorgensen, J. Chandrasekhar, and J. D. Madura, J. Chem. Phys. 79, 926 (1983).

12 E. S. Boek, P. V. Coveney, and N. T. Skipper, J. Amm. Chem. Soc. 117, 12608 (1995).

13 E. S. Boek, P. V. Conveney, and N. T. Skipper, Langmuir 11, 4629 (1995).

14 V. Marry, P. Turq, T. Cartailler, and D. Levesque, J. Chem. Phys. 117, 3454 (2002).

15 D. G. Bounds, Mol. Phys. 54, 1335 (1985).

16 F. Jalilehvand, D. Spangberg, P. Lindqvist-Reis, K. Hermansson, I. Persson, and M. Sandström, J. Amm. Chem. Soc. 123, 431 (2001).

17 N. A. Hewish, G. W. Neilson, and J. E. Enderby, Nature (London) 297, 138 (1982).

18 M. M. Probst, T. Radnai, K. Heinzinger, P. Bopp, and B. M. Rode, J. Phys. Chem. 89, 753 (1985).

19 G. Licheri, G. Piccaluga, and G. Pinna, J. Chem. Phys. 64, 2437 (1976)

${ }^{20}$ C. F. Schwenk, H. H. Loeffler, and B. M. Rode, J. Chem. Phys. 64, 2437 (2001).

21 B. Mehlig, D. W. Heermann, and B. M. Forrest, Phys. Rev.
B. 45, 679 (1992).

22 D. Frenkel and B. Smit, Understanding molecular simulation (Academic, New York, 1996).

23 G. Odriozola, J. F. Aguilar, and J. López-Lemus, J. Chem. Phys. 121, 4266 (2004).

24 M. Tuckerman and B. J. Berne, J. Chem. Phys. 97, 1990 (1992).

25 M. Chávez-Páez, K. Van Workum, L. de Pablo, and J. J. de Pablo, J. Chem. Phys. 114, 1405 (2001).

26 E. J. M. Hensen, T. J. Tambach, A. Bliek, and B. Smit, J. Chem. Phys. 115, 3322 (2001).

27 L. de Pablo, M. L. Chávez, A. K. Sum, and J. J. de Pablo, J. Chem. Phys. 120, 939 (2004).

28 E. J. M. Hensen and B. Smit, J. Phys. Chem. B 106, 12664 (2002).

29 G. Odriozola and F. Guevara, Langmuir 20, 2010 (2004).

30 J. Aquist, J. Phys. Chem. 90, 8021 (1990).

31 E. S. Boek and M. Sprik, J. Phys. Chem. B 107, 3251 (2003).

32 R. W. Mooney, A. G. Keenan, and L. A. Wood, J. Amm. Chem. Soc. 74, 1371 (1952).

33 S. B. Hendricks, R. A. Nelson, and L. T. Alexander, J. Amm. Chem. Soc. 62, 1457 (1940).

34 T. Sato, T. Watanabe, and R. Otsuka, Clays and Clay Miner. 40, 103 (1992).

35 P. G. Slade, S. P. A., and E. W. Radoslovich, Clays and Clay Miner. 33, 51 (1985).

36 K. Tamura, H. Yamada, and H. Nakazawa, Clays and Clay Miner. 48, 400 (2000).

37 J. Cuadros, Am. J. Sci. 297, 829 (1997).

38 A. C. D. Newman, Chemistry of Clays and Clay Minerals (Mineralogical Society, London, 1987).

39 N. T. Skipper, G. D. Williams, A. V. C. de Siqueira, C. Lobban, and A. K. Soper, Clay Minerals 35, 283 (2000).

40 S. Wang, A. F. Koster van Gross, and S. Guggenheim, Geochim. Cosmochim. Ac. 60, 2167 (1996). 